\title{
Heart Rate Variability for Quantification of Autonomic Dysfunction in Fibromyalgia
}

\author{
Jin Ho Kang, $\mathrm{MD}^{1}$, Jong Kyu Kim, $\mathrm{MD}^{1}$, Seok Hyun Hong, $\mathrm{MD}^{1}$, \\ Chang Hyun Lee, $\mathrm{MD}^{1}$, Byoong Yong Choi, $\mathrm{MD}^{2}$
}

Departments of ${ }^{1}$ Physical Medicine and Rehabilitation, ${ }^{2}$ Internal Medicine, Seoul Medical Center, Seoul, Korea

\begin{abstract}
Objective To quantify autonomic dysfunction in fibromyalgia patients compared to healthy controls using heart rate variability (HRV).

Methods Sixteen patients with fibromyalgia and 16 healthy controls were recruited in this case control study. HRV was measured using the time-domain method incorporating the following parameters: total heartbeats, the mean of intervals between consecutive heartbeats (R-R intervals), the standard deviation of normal to normal R-R intervals (SDNN), the square root of the mean squared differences of successive R-R intervals (RMSSD), ratio of SDNN to RMSSD (SDNN/RMSSD), and difference between the longest and shortest R-R interval under different three conditions including normal quiet breathing, rate controlled breathing, and Valsalva maneuver. The severity of autonomic symptoms in the group of patients with fibromyalgia was measured by Composite Autonomic Symptom Scale 31 (COMPASS 31). Then we analyzed the difference between the fibromyalgia and control groups and the correlation between the COMPASS 31 and aforementioned HRV parameters in the study groups.

Results Patients with fibromyalgia had significantly higher SDNN/RMSSD values under both normal quiet breathing and rate controlled breathing compared to controls. Differences between the longest and shortest R-R interval under Valsalva maneuver were also significantly lower in patients with fibromyalgia than in controls. COMPASS 31 score was negatively correlated with SDNN/RMSSD values under rate controlled breathing. Conclusion SDNN/RMSSD is a valuable parameter for autonomic nervous system function and can be used to quantify subjective autonomic symptoms in patients with fibromyalgia.
\end{abstract}

Keywords Fibromyalgia, Autonomic nervous system, Heart rate, Sympathetic nervous system, Parasympathetic nervous system

Received June 4, 2015; Accepted September 1, 2015

Corresponding author: Jong Kyu Kim

Department of Physical Medicine and Rehabilitation, Seoul Medical Center, 156 Sinnae-ro, Jungnang-gu, Seoul 02053, Korea

Tel: +82-2-2276-7474, Fax: +82-2-2276-7486, E-mail: jongkyu.kim.md@ gmail.com

(c) This is an open-access article distributed under the terms of the Creative Commons Attribution Non-Commercial License (http://creativecommons. org/licenses/by-nc/4.0) which permits unrestricted noncommercial use, distribution, and reproduction in any medium, provided the original work is properly cited.

Copyright $\odot 2016$ by Korean Academy of Rehabilitation Medicine

\section{INTRODUCTION}

Fibromyalgia is a syndrome of widespread complaints associated with generalized musculoskeletal pain, fatigue, reduced muscle strength, depressive mood, decreased quality of life and functional limitation [1-4]. Although the etiology of fibromyalgia is unknown and ambiguous, generally it is considered to be a central sensitization and inappropriate diffuse noxious inhibitory 
control system [5,6]. In addition, autonomic dysfunction is a potential mechanism that is associated with pain and psychosocial stress in patients of fibromyalgia [7-10].

Heart rate variability (HRV) is a widely used noninvasive tool to assess the cardiac autonomic nervous system (ANS). Intervals between consecutive heartbeats are not constant, but instead oscillate. And oscillation in cardiac cycles is controlled by sympatho-vagal balance of the ANS. HRV is the measure of inter-beat interval fluctuations in heart rate [11]. Aberrant ANS responses is one biological marker in fibromyalgia and encompasses sympathetic hyperactivity and reduced parasympathetic activity [12-14]. Therefore, patients with fibromyalgia present with blunted sympathetic vascular modulation and impaired cardiac vagal withdrawal to gravitational stress, and consequently reduced orthostatic tolerance [15].

Formerly, variations in heart rate secondary to respiration, head tilt, or Valsalva maneuver were used to measure HRV. But these measurements have some limited reproducibility and objectivity [11]. Power spectral analysis of variations in intervals between consecutive heartbeats (R-R intervals) is now a widely accepted means of evaluating cardiac autonomic regulation. High frequency (HF) values reflect sympathetic activity and low frequency (LF) reflect parasympathetic or both sympathetic and parasympathetic-vagal activity. Furthermore, statistically calculated variables from R-R intervals and differences between R-R intervals suggest that some of these variables seem to correlate with frequency variations $[11,16,17]$.

The primary aim of this study was to compare ANS functions of fibromyalgia and control groups by the timedomain method including classical and statistically calculated variables. Additionally, we evaluated HRV variables for their potential value in the quantification of fibromyalgia by correlation with subjective ANS symptoms assessed by questionnaire.

\section{MATERIALS AND METHODS}

\section{Participants}

Sixteen patients diagnosed with fibromyalgia were recruited from the rheumatologic outpatient service of the Seoul Medical Center. All 16 patients were diagnosed with fibromyalgia by the same rheumatologist and evaluated between June 2014 and August 2014. The sample included 12 women and 4 men whose ages ranged from
37 to 60 years and had been suffering from fibromyalgia for 3 to 20 years. Sixteen healthy gender and age matched individuals that visited same hospital within the same period composed the control group. Each individual in the control group was matched to a patient within 3 years of age. All healthy controls reported being pain free at time of testing and were not taking any analgesics or antiinflammatory drugs.

In the first visit, the rheumatologist screened patients who had previously been diagnosed with fibromyalgia by medical history questionnaire to determine eligibility for enrollment in the study. Next, baseline characteristics and body mass index were recorded for the members of the study group. Fibromyalgia Impact Questionnaire (FIQ) and Numeric Pain Intensity Score (NPIS) ranging from 0 (no pain) to 100 (most intense pain) were also examined. Exclusion criteria were current smokers; blood pressure greater than $160 / 100 \mathrm{mmHg}$; central nervous system, coronary artery, peripheral vascular diseases; and diabetes, renal, adrenal, pituitary and thyroid disorders.

ANS measurements were taken in the second visit. It was requested that patients visit the hospital in the afternoon and to refrain from caffeine and alcohol for at least 12 hours and vigorous physical activity for at least 24 hours prior to ANS measurement. All investigations took approximately 20 minutes for each subject excluding a resting period.

\section{Autonomic symptom measurements}

The Composite Autonomic Symptom Scale (COMPASS) is a questionnaire designed to evaluate the severity and distribution of autonomic symptoms of patients with autonomic dysfunction [18]. Although COMPASS is a well-established tool for assessment of ANS function, it is complex and contains inherent inconsistencies. Therefore, the new instrument COMPASS 31 was developed with reduced redundant content and a new, total of 31 questions in 6 domains (orthostatic intolerance, 4 items; vasomotor, 3 items; secretomotor, 4 items; gastrointestinal, 8 items; constipation, 4 items; bladder, 3 items; pupillomotor, 5 items). COMPASS 31 is a refined, more internally consistent, quantitative measure of autonomic symptoms [19].

\section{Heart rate variability}

Continuous electrocardiogram (ECG) was recorded by 
three-lead electrocardiograph using Sierra Wave EMG system (Cadwell Laboratories Inc., Kennewick, WA, USA). Each subject laid in a supine position in a quiet room for at least 10 minutes prior to the recording. The first recording was started silently after instructing the subject to breathe normally. The second recording was taken after providing an explanation of controlled breathing rate with verbal instructions to take 6 breaths per minute. All recordings were taken at specific times in the afternoon.

Detection of QRS complexes and intervals between consecutive heartbeats (R-R intervals) were measured by a single examiner using the same electromyography device. The selected time domain parameters were total heartbeats (THB); the mean of R-R intervals (MRR); the standard deviation of normal to normal R-R intervals (SDNN); the square root of the mean squared differences of successive R-R intervals (RMSSD); and ratio of SDNN to RMSSD (SDNN/RMSSD) [10,11,20,21]. All timedomain variables of normal quite breathing and rate controlled breathing were calculated using Microsoft Excel 2010 (Microsoft, Redmond, WA, USA) by the following detailed equations $[11,21]$ :

$$
\begin{gathered}
\text { THB }=\mathrm{N} \\
\text { MRR }=\bar{I}=\frac{1}{\mathrm{~N}-1} \sum_{n=2}^{N} I(n) \\
\mathrm{SDNN}=\sqrt{\frac{1}{\mathrm{~N}-1} \sum_{n=2}^{N}[I(n)-\bar{I}]^{2}} \\
\mathrm{RMSSD}=\sqrt{\frac{1}{\mathrm{~N}-2} \sum_{n=3}^{N}[I(n)-I(n-1)]^{2}}
\end{gathered}
$$

Additionally, simple time-domain variables of HRV calculated from the difference between the longest and shortest R-R interval under different three conditions including normal breathing, controlled breathing, and Valsalva maneuver were obtained as traditionally used values [11].

\section{Statistical analysis}

Mann-Whitney U tests were used to assess group differences between fibromyalgia versus control. The ShapiroWilk test and Kolmogorov-Smirnov test were used to assess whether the data showed a normal distribution. In the fibromyalgia group, the associations between subjective symptoms including COMPASS 31, FIQ, NPIS, and HRV data were estimated by Pearson correlation and linear regression analysis in which data showed normal distribution. $\mathrm{R}$ ver. 3.2.0 software ( $\mathrm{R}$ Development Core Team) was used for statistical analysis. All data are reported as mean \pm standard deviation. Significance was considered when $\mathrm{p}$-value was less than 0.05 .

\section{RESULTS}

Table 1 summarizes baseline characteristics of all participants. There were no significant differences between the two groups in age, sex, height, weight, and body mass index.

R-R intervals between two groups showed no significant difference under rate controlled breathing and Valsalva maneuver. Reference values of R-R intervals in our lab are represented in Table 2.

Patients with fibromyalgia had significantly higher $(p<0.05)$ SDNN/RMSSD values under both normal quiet breathing and rate controlled breathing compared to controls. Difference between the longest and shortest R-R interval under Valsalva maneuver were also significantly $(\mathrm{p}<0.05)$ lower in patients with fibromyalgia than in con-

Table 1. Baseline demographic and clinical characteristics of the participants

\begin{tabular}{lccc}
\hline & Fibromyalgia $(\mathbf{n}=\mathbf{1 6})$ & Control $(\mathbf{n}=\mathbf{1 6})$ & p-value \\
\hline Age $(\mathrm{yr})$ & $49.8 \pm 7.4$ & $49.9 \pm 7.6$ & 0.806 \\
Sex & & & 1.000 \\
Male & $4(25.0)$ & $4(25.0)$ & \\
Female & $12(75.0)$ & $12(75.0)$ & \\
Height $(\mathrm{cm})$ & $157.4 \pm 6.4$ & $160.4 \pm 6.4$ & 0.212 \\
Weight $(\mathrm{kg})$ & $63.7 \pm 9.7$ & $59.2 \pm 8.9$ & 0.193 \\
Body mass index $\left(\mathrm{kg} / \mathrm{m}^{2}\right)$ & $25.7 \pm 3.6$ & $23.6 \pm 2.0$ & 0.063 \\
\hline
\end{tabular}

Values are presented as mean \pm standard deviation or number (\%). 
trols. There was no significant difference in THB, MRR, SDNN and RMSSD under both normal quiet breathing and rate controlled breathing as well as difference of

Table 2. Reference values of R-R interval difference

\begin{tabular}{|lcc|}
\hline & $\begin{array}{c}\text { Age } \\
\text { (yr) }\end{array}$ & $\begin{array}{c}\text { R-R interval } \\
\text { difference }(\mathbf{m s})\end{array}$ \\
\hline Deep breathing & $16-20$ & $>1.23$ \\
& $21-25$ & $>1.20$ \\
& $26-30$ & $>1.18$ \\
& $31-35$ & $>1.16$ \\
\hline $36-40$ & $>1.14$ \\
\hline & $41-45$ & $>1.12$ \\
& $46-50$ & $>1.11$ \\
\hline & $51-55$ & $>1.09$ \\
& $56-60$ & $>1.08$ \\
\hline Valsalva & $61-65$ & $>1.07$ \\
& $66-75$ & $>1.06$ \\
\hline & $76-80$ & $>10.5$ \\
\hline Standing & $10-40$ & $>1.5$ \\
\hline & $41-50$ & $>1.45$ \\
\hline & $51-60$ & $>1.40$ \\
\hline
\end{tabular}

R-R interval under normal quiet breathing and rate controlled breathing (Table 2).

In Shapiro-Wilk test, SDNN/RMSSD under normal quiet breathing (NB ratio) and rate controlled breathing ( $\mathrm{CB}$ ratio) showed normal distribution. NPIS and FIQ also showed normal distribution with Kolmogorov-Smirnov test. Linear regression was used to evaluate association between COMPASS 31 and SDNN/RMSSD under both conditions. COMPASS 31 score revealed a significant negative correlation with SDNN/RMSSD under rate controlled breathing ( $\mathrm{y}=-10.562 \mathrm{x}+61.629, \mathrm{R}=0.546, \mathrm{p}=0.029$ ), but not correlated with SDNN/RMSSD under normal breathing (Fig. 1). FIQ and NPIS score showed no significant correlation with SDNN/RMSSD under both conditions (FIQ and NB ratio, $\mathrm{p}=0.84$; FIQ and $\mathrm{CB}$ ratio, $\mathrm{p}=0.96$; NPIS and NB ratio, $\mathrm{p}=0.68$; NPIS and $\mathrm{CB}$ ratio, $\mathrm{p}=0.61$ ) (Table 3).

\section{DISCUSSION}

The nature of autonomic function in patients with fibromyalgia shows sympathetic hyperactivity and parasympathetic hypoactivity [12-14]. Following the benchmark paper of ANS measurement [11], there were many studies suggesting fibromyalgia was associated with

Table 3. Comparison of autonomic function between fibromyalgia and control group

\begin{tabular}{|lccc|}
\hline & Fibromyalgia $(\mathbf{n}=\mathbf{1 6})$ & Control $(\mathbf{n}=\mathbf{1 6})$ & p-value \\
\hline COMPASS 31 & $43.94 \pm 8.92$ & - & \\
\hline FIQ & $80.4 \pm 9.2$ & - & \\
\hline NPIS & $78.5 \pm 13.9$ & - & \\
\hline Normal quiet breathing & & & 0.970 \\
\hline SDNN & $25.73 \pm 20.23$ & $23.51 \pm 13.51$ & 0.243 \\
\hline RMSSD & $21.46 \pm 19.14$ & $21.61 \pm 9.45$ & $0.015^{*}$ \\
\hline SDNN/RMSSD & $1.36 \pm 0.38$ & $1.07 \pm 0.20$ & \\
Rate controlled breathing & & & 0.274 \\
\hline SDNN & $62.12 \pm 28.11$ & $51.72 \pm 21.77$ & 0.678 \\
\hline RMSSD & $41.67 \pm 24.98$ & $43.00 \pm 21.23$ & $0.003^{* *}$ \\
\hline SDNN/RMSSD & $1.68 \pm 0.46$ & $1.25 \pm 0.20$ & $0.001^{* *}$ \\
\hline VALSALVA & $1.42 \pm 0.23$ & $1.48 \pm 0.06$ & \\
\hline
\end{tabular}

Values are presented as mean \pm standard deviation.

COMPASS 31, Composite Autonomic Symptom Scale 31; FIQ, Fibromyalgia Impact Questionnaire; NPIS, Numeric Pain Intensity Score; SDNN, standard deviation of normal to normal R-R intervals; RMSSD, square root of the mean squared differences of successive R-R intervals; SDNN/RMSSD, ratio of SDNN to RMSSD; VALSALVA, difference between the longest and shortest R-R interval under Valsalva maneuver.

${ }^{*} \mathrm{p}<0.05,{ }^{* *} \mathrm{p}<0.01$. 
(A)

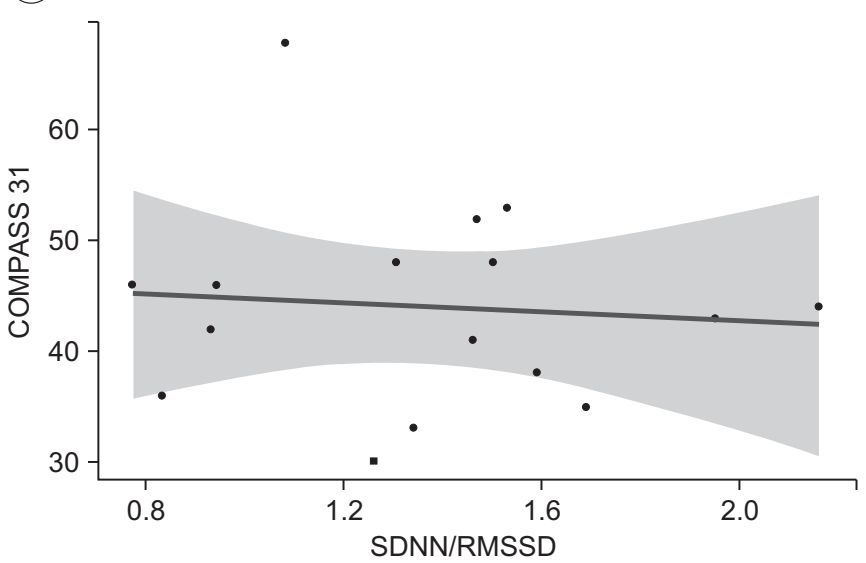

(B)

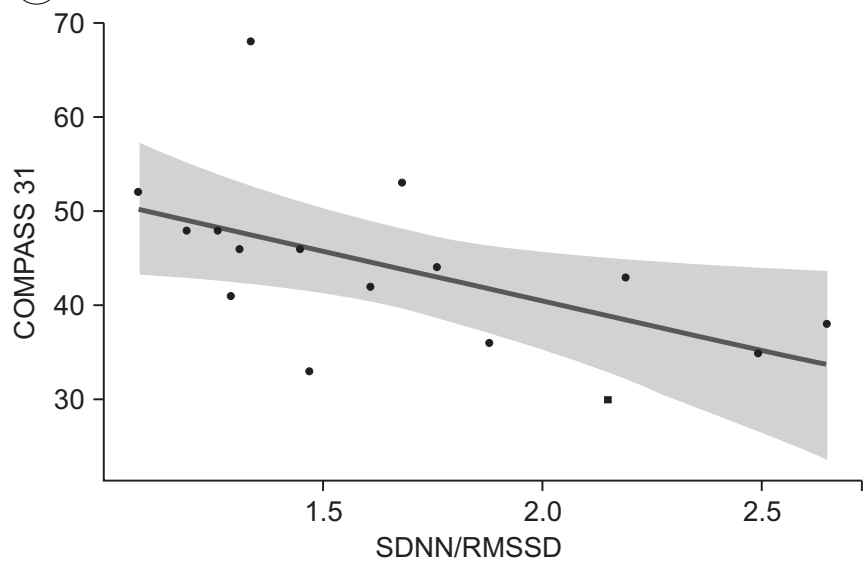

Fig. 1. Correlation between COMPASS 31 and ratio of SDNN to RMSSD (SDNN/RMSSD) under rate controlled breathing. Linear regression analysis was used to determine the relation between COMPASS 31 (y-axis) and SDNN/RMSSD ( $\mathrm{x}$-axis). (A) No significant correlation under normal quiet breathing $(\mathrm{p}=0.756)$. (B) Significant negative correlation was revealed under rate controlled breathing $(\mathrm{y}=-10.562 \mathrm{x}+61.629, \mathrm{R}=0.546, \mathrm{p}=0.029)$. COMPASS 31, Composite Autonomic Symptom Scale 31; SDNN, standard deviation of normal to normal R-R intervals; RMSSD, square root of the mean squared differences of successive R-R intervals.

reduced parasympathetic activity by using HF power in the frequency-domain method, defined as $0.15-0.40 \mathrm{~Hz}$ and the time-domain method, RMSSD $[9,10,20]$. The major contributor to the HF component is efferent vagal activity. Previous studies have demonstrated that the HF component changes after electrical vagal stimulation, muscarinic receptor blockade, and vagotomy [11]. SDNN reflects all the cyclic components responsible for variability during the recording period. LF reflects a combination of sympathetic and parasympathetic activity $[11,16]$. A previous study suggests that SDNN is correlated with LF power and RMSSD is correlated with HF power during sleep in men [17]. However, significant differences in aforementioned variables including time and frequency-domain measurements have not achieved consensus because of discrepancies in methodological quality [10]. In our study, there was no significant difference in SDNN and RMSSD between fibromyalgia patients and the control group. This result was consistent with previous studies showing no significant differences in LF, SDNN, HF or RMSSD between each group [10,20]. Moreover, recently studies suggested that the time-domain SDNN or RMSSD seemed to have no correlation with the frequency-domain, LF or HF. RMSSD was not significantly affected by changes in respiration [22], but HF power was associated with respiration [23]. Furthermore, correlations were suggested between SDNN and other variables including total, ultra-low or very low frequencies, but they were controversial $[24,25]$.

Although there was some initial disagreement, the ratio of LF to HF (LF/HF) has gained wide acceptance as a means of demonstrating balance between sympathetic and parasympathetic activities; increases in LF/HF are assumed to reflect a shift toward sympathetic dominance and decreases in LF/HF are assumed to reflect a shift towards parasympathetic dominance $[26,27]$. Furthermore, one animal study reported that rats with sympathectomy showed increased HF to LF ratio and rats with vagotomy showed reduced HF to LF ratio [28]. Yet other studies showed that SDNN/RMSSD was a good representation of LF/HF in both study patients and normal healthy controls and had a correlation coefficient of 0.90 in resting supine position $[21,29]$. In our study, the fibromyalgia group had significantly higher SDNN/RMSSD values than the control group regardless of breathing rate. As previously noted, increased LF/HF corresponds with a more sympathetic or less parasympathetic autonomic state in patients with fibromyalgia; this result is consistent with the nature of autonomic function in fibromyalgia. We expected RMSSD and SDNN to reflect autonomic status and to observe lower RMSSD or higher SDNN in fibromyalgia patients. However, there was no significant difference in RMSSD or SDNN between the two groups; only SDNN/RMSSD was significantly higher in the fibro- 
myalgia group when breathing rate was both controlled and not controlled. If SDNN/RMSSD is an appropriate representation of LF/HF, higher SDNN/RMSSD in fibromyalgia compared with healthy controls is consistent with previous studies $[27,30]$.

COMPASS is a questionnaire-based quantitative measurement of autonomic symptoms. In patients with fibromyalgia, highly significant correlation was observed between COMPASS and FIQ ( $\mathrm{R}=0.5)$ [18] or fatigue impact scale $(\mathrm{R}=0.4)$ [31]. In our study, only breathing ratecontrolled SDNN/RMSSD was significantly correlated with COMPASS 31 ( $\mathrm{y}=-10.562 \mathrm{x}+61.629, \mathrm{R}=0.546, \mathrm{p}=0.029$ ). Therefore, controlled breathing rate may be a means of quantifying subjective symptoms because a subject's cardiovascular activity is influenced by individual different breathing rate in normal quiet breathing. The difference between the longest and shortest R-R interval under Valsalva maneuver did not show normal distribution and did not correlate with COMPASS 31 .

Chronic widespread pain and tenderness is a major problem encountered by patients with fibromyalgia, which has disastrous effects on quality of life and contributes to socio-economic losses [32]. Although the pathophysiology of pain in fibromyalgia is still unclear, there are two different theories for the pain mechanism associated with the condition. Previously, altered central processing of nociceptive information was the dominant concept to explain the pathophysiology of fibromyalgia $[6,33]$. In the presence of nociceptive stimulation, descending inhibitory efferents arising from brainstem nuclei modulate the pain threshold to reduced pain sensitivity. These brainstem nuclei (including periaqueductal gray, locus coeruleus, rosto-ventromedial medulla, and nucleus tractus solitaries) also play a key role in ANS control. Moreover, patients with fibromyalgia showed increased LF and reduced HF in response to mild pain, whereas healthy controls showed reduced LF and increased HF [33]. As many studies have noted dysfunction of pain-modulating responses in patients with fibromyalgia, this concept has become a major theory of the pathophysiology of fibromyalgia $[6,33,34]$.

However, recently studies have found impaired small fiber function in patients with fibromyalgia $[35,36]$. In patients with fibromyalgia, these studies have reported reduced epidermal unmyelinated nerve fiber (C-fiber) density upon skin biopsy; abnormalities in pain-related evoked potentials and quantitative sensory testing; small fiber neuropathy profiles via questionnaire [36]; and abnormalities in C-fiber action potentials by microneurography [35]. Therefore, there is now increasing evidence of small fiber neuropathic tendencies in fibromyalgia. In this study, all patients with fibromyalgia complained of severe pain intensity score $(80.44 \pm 12.86)$ and FIQ score (81.99 \pm 8.85$)$. FIQ score and pain intensity score were significantly correlated with one another, but there was no significant correlation between FIQ or pain and any other variables including COMPASS 31 and SDNN/RMSSD. This result may be explained by fibromyalgia patients' life-style being more heavily impacted by their pain intensity rather than their autonomic symptoms. Furthermore, contributors to the pain of fibromyalgia include not only central pain inhibitory dysfunction accompanied by autonomic abnormality, but also other potential mechanisms such as small fiber neuropathy.

Our study had several limitations. First, the number of subjects in both groups was relatively small. However, because all subjects were evaluated within a relatively short period of 3 months between June and August and at a specific time in the afternoon, this was intended to minimize bias from seasonal or daily fluctuations. To our knowledge, reference values of SDNN/RMSSD are not established. Our study demonstrated that fibromyalgia patients showed different values from healthy controls, but we could not present normal or cutoff values of SDNN/ RMSSD because of the small sample size. To suggest reference values of SDNN/RMSSD, a larger study with age/ gender specific values is needed. Second, we assessed only time-domain measurement. There are many studies using frequency-domain measurement but analysis of frequency requires a specific device. Therefore, we designed this study with time-domain measurement to evaluate the ANS dysfunction of fibromyalgia patients. Our hypothesis was based on previous studies using frequency analysis with more widely accepted results; however, correlation between each value of time-domain and frequency-domain remained controversial $[11,16,17]$. Third, every assessor was blinded to the results of each evaluation and calculation, but we did not complete blind trials for the patient and control groups.

Lastly, at the time of the study, our patients had taken various medications to relieve the symptoms of fibromyalgia: 9 subjects were taking anticonvulsants (valproic 
acid, pregabalin, or topiramate); 2 , selective serotonin reuptake inhibitors (paroxetine); 5 , serotonin-norepinephrine reuptake inhibitors (milnacipran or duloxetine); and 5, tricyclic antidepressants (nortriptyline). We did not restrict patients from using these drugs, because patients had a history of chronic use of the medications (over 2 months) and were subsequently apprehensive of experiencing pain after discontinuation their respective medications. In a previous meta-analysis study, there was a trend of higher LF values in subjects taking anticonvulsants [37]. In addition, serotonin-norepinephrine reuptake inhibitors and tricyclic antidepressants were associated with lower SDNN, LF and HF, but selective serotonin reuptake inhibitors were not significantly associated with HRV $[38,39]$. These medications may have influenced the HRV of the study group. In our study, within the fibromyalgia group there was no significant difference in COMPASS 31 score and SDNN/RMSSD under both conditions whether subjects taking medication or not in any of the aforementioned 4 drug categories.

Nonetheless, this study shows that SDNN/RMSSD is a valuable parameter for evaluation of ANS dysfunction in fibromyalgia patients.

In conclusion, SDNN/RMSSD under rate-controlled breathing is significantly higher in patients with fibromyalgia and has a strong correlation with COMPASS 31. SDNN/RMSSD should be considered a valuable parameter of time-domain measurement of ANS function; it is also a useful parameter to quantify subjective symptoms in fibromyalgia. Because of the small sample size in this study, a larger, well-constructed study is needed.

\section{CONFLICT OF INTEREST}

No potential conflict of interest relevant to this article was reported.

\section{REFERENCES}

1. Bennett RM, Jones J, Turk DC, Russell IJ, Matallana L. An internet survey of 2,596 people with fibromyalgia. BMC Musculoskelet Disord 2007;8:27.

2. Cohen H, Neumann L, Shore M, Amir M, Cassuto Y, Buskila D. Autonomic dysfunction in patients with fibromyalgia: application of power spectral analysis of heart rate variability. Semin Arthritis Rheum
2000;29:217-27.

3. Hoffman DL, Dukes EM. The health status burden of people with fibromyalgia: a review of studies that assessed health status with the SF-36 or the SF-12. Int J Clin Pract 2008;62:115-26.

4. Kurtze N, Gundersen KT, Svebak S. Quality of life, functional disability and lifestyle among subgroups of fibromyalgia patients: the significance of anxiety and depression. Br J Med Psychol 1999;72(Pt 4):471-84.

5. Clauw DJ, Arnold LM, McCarberg BH; FibroCollaborative. The science of fibromyalgia. Mayo Clin Proc 2011;86:907-11.

6. Lautenbacher S, Rollman GB. Possible deficiencies of pain modulation in fibromyalgia. Clin J Pain 1997;13:189-96.

7. Buskila D. Developments in the scientific and clinical understanding of fibromyalgia. Arthritis Res Ther 2009;11:242.

8. Figueroa A, Kingsley JD, McMillan V, Panton LB. Resistance exercise training improves heart rate variability in women with fibromyalgia. Clin Physiol Funct Imaging 2008;28:49-54.

9. Kulshreshtha P, Deepak KK. Autonomic nervous system profile in fibromyalgia patients and its modulation by exercise: a mini review. Clin Physiol Funct Imaging 2013;33:83-91.

10. Tak LM, Riese H, de Bock GH, Manoharan A, Kok IC, Rosmalen JG. As good as it gets? A meta-analysis and systematic review of methodological quality of heart rate variability studies in functional somatic disorders. Biol Psychol 2009;82:101-10.

11. Task Force of the European Society of Cardiology and the North American Society of Pacing and Electrophysiology. Heart rate variability: standards of measurement, physiological interpretation, and clinical use. Eur Heart J 1996;17:354-81.

12. Cohen H, Neumann L, Alhosshle A, Kotler M, AbuShakra M, Buskila D. Abnormal sympathovagal balance in men with fibromyalgia. J Rheumatol 2001;28: 581-9.

13. Martinez-Lavin M, Hermosillo AG, Rosas M, Soto ME. Circadian studies of autonomic nervous balance in patients with fibromyalgia: a heart rate variability analysis. Arthritis Rheum 1998;41:1966-71.

14. Raj SR, Brouillard D, Simpson CS, Hopman WM, Abdollah H. Dysautonomia among patients with fi- 
bromyalgia: a noninvasive assessment. J Rheumatol 2000;27:2660-5.

15. Furlan R, Colombo S, Perego F, Atzeni F, Diana A, Barbic F, et al. Abnormalities of cardiovascular neural control and reduced orthostatic tolerance in patients with primary fibromyalgia. J Rheumatol 2005;32:178793.

16. Cevese A, Gulli G, Polati E, Gottin L, Grasso R. Baroreflex and oscillation of heart period at $0.1 \mathrm{~Hz}$ studied by alpha-blockade and cross-spectral analysis in healthy humans. J Physiol 2001;531(Pt 1):235-44.

17. Otzenberger H, Gronfier C, Simon C, Charloux A, Ehrhart J, Piquard F, et al. Dynamic heart rate variability: a tool for exploring sympathovagal balance continuously during sleep in men. Am J Physiol 1998;275(3 Pt 2):H946-50.

18. Solano C, Martinez A, Becerril L, Vargas A, Figueroa J, Navarro C, et al. Autonomic dysfunction in fibromyalgia assessed by the Composite Autonomic Symptoms Scale (COMPASS). J Clin Rheumatol 2009;15:172-6.

19. Sletten DM, Suarez GA, Low PA, Mandrekar J, Singer W. COMPASS 31: a refined and abbreviated Composite Autonomic Symptom Score. Mayo Clin Proc 2012;87:1196-201.

20. Meeus M, Goubert D, De Backer F, Struyf F, Hermans L, Coppieters I, et al. Heart rate variability in patients with fibromyalgia and patients with chronic fatigue syndrome: a systematic review. Semin Arthritis Rheum 2013;43:279-87.

21. Wang HM, Huang SC. SDNN/RMSSD as a surrogate for LF/HF: a revised investigation. Model Simul Eng 2012;2012:1-8.

22. Penttila J, Helminen A, Jartti T, Kuusela T, Huikuri HV, Tulppo MP, et al. Time domain, geometrical and frequency domain analysis of cardiac vagal outflow: effects of various respiratory patterns. Clin Physiol 2001;21:365-76.

23. Berntson GG, Lozano DL, Chen YJ. Filter properties of root mean square successive difference (RMSSD) for heart rate. Psychophysiology 2005;42:246-52.

24. Bigger JT Jr, Fleiss JL, Steinman RC, Rolnitzky LM, Kleiger RE, Rottman JN. Correlations among time and frequency domain measures of heart period variability two weeks after acute myocardial infarction. Am J Cardiol 1992;69:891-8.

25. Calvert CA, Wall TM. Correlations among time and frequency measures of heart rate variability recorded by use of a Holter monitor in overtly healthy Doberman pinschers with and without echocardiographic evidence of dilated cardiomyopathy. Am J Vet Res 2001;62:1787-92.

26. Balocchi R, Cantini F, Varanini M, Raimondi G, Legramante JM, Macerata A. Revisiting the potential of time-domain indexes in short-term HRV analysis. Biomed Tech (Berl) 2006;51:190-3.

27. Malliani A, Pagani M, Lombardi F, Cerutti S. Cardiovascular neural regulation explored in the frequency domain. Circulation 1991;84:482-92.

28. Yeo HS, Im JJ, Park HT. An analysis of HRV spectrum for observing ANS variations caused by sympathectomy and vagotomy. Korean J Phys Anthropol 1999;12:289-96.

29. Sollers JJ 3rd, Buchanan TW, Mowrer SM, Hill LK, Thayer JF. Comparison of the ratio of the standard deviation of the R-R interval and the root mean squared successive differences (SD/rMSSD) to the low frequency-to-high frequency (LF/HF) ratio in a patient population and normal healthy controls. Biomed Sci Instrum 2007;43:158-63.

30. Pagani M, Lombardi F, Guzzetti S, Sandrone G, Rimoldi O, Malfatto G, et al. Power spectral density of heart rate variability as an index of sympatho-vagal interaction in normal and hypertensive subjects. J Hypertens Suppl 1984;2:S383-5.

31. Newton JL, Okonkwo O, Sutcliffe K, Seth A, Shin J, Jones DE. Symptoms of autonomic dysfunction in chronic fatigue syndrome. QJM 2007;100:519-26.

32. Kim SK, Kim SH, Lee CK, Lee HS, Lee SH, Park YB, et al. Effect of fibromyalgia syndrome on the healthrelated quality of life and economic burden in Korea. Rheumatology (Oxford) 2013;52:311-20.

33. Chalaye P, Goffaux P, Bourgault P, Lafrenaye S, Devroede $G$, Watier $A$, et al. Comparing pain modulation and autonomic responses in fibromyalgia and irritable bowel syndrome patients. Clin J Pain 2012;28:51926.

34. Woolf CJ. Central sensitization: implications for the diagnosis and treatment of pain. Pain 2011;152(3 Suppl):S2-15.

35. Serra J, Collado A, Sola R, Antonelli F, Torres X, Salgueiro $\mathrm{M}$, et al. Hyperexcitable $\mathrm{C}$ nociceptors in fibromyalgia. Ann Neurol 2014;75:196-208. 
36. Uceyler N, Zeller D, Kahn AK, Kewenig S, KittelSchneider S, Schmid A, et al. Small fibre pathology in patients with fibromyalgia syndrome. Brain 2013;136(Pt 6):1857-67.

37. Lotufo PA, Valiengo L, Bensenor IM, Brunoni AR. A systematic review and meta-analysis of heart rate variability in epilepsy and antiepileptic drugs. Epilepsia 2012;53:272-82.
38. Kemp AH, Quintana DS, Gray MA, Felmingham KL, Brown K, Gatt JM. Impact of depression and antidepressant treatment on heart rate variability: a review and meta-analysis. Biol Psychiatry 2010;67:1067-74.

39. Yeragani VK, Pesce V, Jayaraman A, Roose S. Major depression with ischemic heart disease: effects of paroxetine and nortriptyline on long-term heart rate variability measures. Biol Psychiatry 2002;52:418-29. 